\title{
Una experiencia en Chile de trabajo grupal con dirigentes de organizaciones sociales
}

\section{A group work experience in Chile with leaders of social organizations}

\author{
Clàudia TURRÓ ORTEGA* \\ M. - Teresa RAMÍREZ CORVERA \\ Mariane KRAUSE JACOB

\section{RESUMEN}

Ante la necesidad de apoyo expresada por vecinos de la comunidad chilena de La Victoria a los organismos gubernamentales, por situaciones de inseguridad ligadas al microtráfico, surge la intervención psicológico-comunitaria que enmarca el trabajo grupal presentado en el artículo. Esta intervención grupal tuvo lugar durante los años 2003 y 2004 y se realizó con representantes de distintas organizaciones sociales de La Victoria, con el objetivo de formar un equipo de trabajo, denominado Coordinadora de organizaciones sociales, capaz de llevar a cabo de manera eficiente los proyectos comunitarios que se planteara. El proceso grupal fue facilitado por dos psicólogas y se utilizó como metodologia, principalmente, técnicas participativas de educación popular, las cuales se dirigieron a potenciar las habilidades socio-emocionales y de tarea de los participantes del grupo. En último lugar se presentan los resultados, que dicen relación con los objetivos específicos propuestos para la intervención grupal, así como los logros y algunas reflexiones en torno a la experiencia. Al respecto, se destaca el anclaje de la realidad cultural-histórica de La Victoria en algunos comportamientos grupales, la importancia del proceso de inserción en la comunidad y la reflexión sobre el quehacer profesional del equipo de trabajo.

\footnotetext{
* Universidad Católica de Chile.
} 
Una experiencia en Chile de trabajo grupal con dirigentes de organizaciones sociales

\title{
PALABRAS CLAVE
}

Participación social, Comunidad, Intervención grupal, Equipo de trabajo.

\begin{abstract}
The psychological-communitarian intervention which has framed the group-work presented in this article, emerged from the need of support expressed by the neighbours of the Chilean community of La Victoria to the governmental bodies, due to insecurity situations bound to micro traffic. This group-intervention took place during 2003 and 2004 with representatives of different social organizations of La Victoria. The aim of the intervention was to form a work team called "Coordinator of social organizations" that was able to develop communitarian projects to maximize the social participation of the population. This process was conducted by two psychologists who mainly used popular education participation techniques as the work methodology. These techniques were addressed to strengthen the socio-emotional abilities and the task abilities of the members of the group. In the end the results show relation with the specific aims proposed for the group-intervention, the achievements, and various reflections around the experience. Especially mentioned are the anchorage of the cultural and historical reality of the community La Victoria in some groupbehaviours, the importance of the process of insertion in the community, and the reflection about the tasks of the professional team.
\end{abstract}

\section{KEY WORDS}

Social participation, Community, Intervention group, Work team. 


\section{CONTEXTO DE APLICACIÓN}

$\mathrm{El}$ artículo presenta parte de la segunda etapa de una intervención comunitaria llevada a cabo en Chile, concretamente en la población ${ }^{1}$ La Victoria, entre los años 2002 y 2004, por un equipo de siete profesionales. Esta intervención surgió en el marco del Programa Barrios Seguros, implementado y financiado por la Subsecretaría del Interior, desde la División de Seguridad Ciudadana del gobierno chileno. Dicho programa tuvo como finalidad dirigirse a distintas poblaciones de Santiago de Chile, aquejadas por problemas de violencia y microtráfico, con la voluntad de involucrar a varios actores sociales, en pro de construir una comunidad más segura, además de enfrentar el problema de la delincuencia desde una óptica multidimensional (policial, legal, psicosocial, ambiental, educacional y de salud). El equipo, responsable de diseñar y ejecutar la intervención en La Victoria, estuvo conformado por seis psicólogos comunitarios y un comunicador social, vinculados a la Pontificia Universidad Católica de Chile, entidad que prestó sus servicios profesionales a las autoridades estatales.

La Victoria fue la segunda población donde se llevó a cabo el Programa Barrios Seguros, debido a la demanda interpuesta por la Junta de Vecinos a la Subsecretaría del Interior, para solucionar los problemas de inseguridad, derivados del consumo y tráfico de drogas. Es decir, la misma comunidad fue la responsable de la puesta en funcionamiento del mencio- nado programa, manifestando una postura activa frente a dicha problemática. Esta actitud se relacionaba, de algún modo, con el carácter empoderado de La Victoria, explicado por su historia, la que se expone brevemente a continuación, para otorgar una mayor comprensión del contexto donde se realizó la intervención.

La Victoria nació el año 1957 a partir de una toma de terrenos ${ }^{2}$, la primera realizada en Latinoamérica, por un grupo de familias provenientes de distintas poblaciones "callampas" ${ }^{3}$ de Santiago (Paiva, 1989). A pesar de ser éste un hecho que señala la condición de extrema pobreza de sus habitantes, también demuestra la capacidad de darse a sí mismos formas de organización propias (Cortés, 2003). En el mismo sentido, muchos habitantes de esta población, aún cuando estuvieron en condiciones económicas y sociopolíticas adversas (pobreza y represión durante la dictadura), desarrollaron una serie de acciones sociales a partir de la participación ciudadana y en organizaciones comunitarias, que les permitieron enfrentar estas condiciones. Sin embargo, desde el año 1990, este accionar como población fue diluyéndose, producto del cambio en Chile de régimen político (el objetivo combativo de las organizaciones perdía sentido en un sistema de transición democrática ${ }^{4}$ y del microtráfico que se inserta en la población.

A raíz de este último aspecto, acrecentado por los años, se perfiló la problemática general abordada en la intervención

\footnotetext{
${ }^{1}$ Población, en el contexto chileno, hace referencia a un asentamiento urbano cuya construcción responde a planes de vivienda social, iniciados por el gobierno aproximadamente en los años '50, para hacer frente al déficit habitacional.

${ }^{2}$ Ocupación de terrenos baldíos realizada de forma coordinada por organizaciones de pobladores sin casa.

${ }^{3}$ Nombre que recibieron en Chile las poblaciones construidas de material ligero (cartón y plástico).

${ }^{4}$ Esta transición se refiere al proceso politico que tuvo lugar en Chile, posterior a la dictadura militar de Augusto Pinochet.
} 
a principios del 2002. Es decir, la violencia y el temor, generados por el microtráfico y la delincuencia, implicaron un repliegue de la participación y la organización comunitaria en La Victoria, constituyendo un círculo vicioso, ya que la ruptura de los vínculos sociales aumentó la sensación subjetiva de amenaza y, a la vez, dejó el territorio más vulnerable para el aumento de la delincuencia, la violencia y el microtráfico ${ }^{5}$. Estos dos efectos, según algunos autores, pueden revertirse en la medida que las organizaciones comunitarias y sus actores estén bien coordinados interna y externamente, favoreciendo la sensación de seguridad de los barrios (Gotzens, 1993; Chalom, Léonard, Vanderschueren y Vézina, 2001). De forma coherente con lo planteado, se planteó como objetivo general de la intervención psicológico-comunitaria potenciar la participación de los habitantes de la población La Victoria, tanto a nivel individual como organizacional, en las actividades implementadas por ellos mismos en la población.

Puesto que la población La Victoria se constituyó como sujeto y objeto demasiado amplio para nuestro quehacer comunitario, se hizo necesario trabajar a distintos niveles con la comunidad, a fin de conseguir una efectiva participación de, al menos, parte de ella. A partir del diagnóstico realizado durante parte del año 2002 (mediante grupos de discusión, entrevistas semi-estructuradas a personas clave y una encuesta aplicada por los mismos pobladores en el seno de sus familias), se tomó la decisión de trabajar con dos grupos de la comunidad: delegadas de cuadra ${ }^{6}$ (representantes electas por cada cuadra de la población) y organizaciones sociales. Si bien todos los miembros del equipo de psicólogos participaron de la planificación y reflexión de la intervención psicológico-comunitaria, aquel se dividió en dos subequipos; de este modo, cada uno de estos trabajó específicamente con uno de los dos grupos antes mencionados.

Siendo que el foco de la intervención expuesto en el artículo dice relación con las organizaciones sociales, se considera pertinente señalar unos antecedentes previos al trabajo grupal específico que será descrito posteriormente.

La intervención con las organizaciones sociales comenzó el 2002, con la realización de una actividad comunitaria propuesta por los mismos pobladores: la $1^{\text {a }}$ Feria de organizaciones sociales. A fin de planificarla e implementarla, se conformó un equipo mixto formado por algunos miembros del equipo interventor y varios pobladores. Durante el desarrollo de la Feria, de un día de duración, las organizaciones expusieron, a través de stands y carteles, sus objetivos, acciones e historia vinculada con La Victoria. A través de dicha actividad, se fomentó por parte de organizaciones sociales, en concreto, y comunidad, en general, la apropiación del espacio público de la población relegado desde algún tiempo al microtráfico. Asimismo, se favoreció la vinculación de los vecinos a algunas organizaciones para una futura participación a corto y mediano plazo.

Consecutivamente a esta primera etapa de intervención, se realizó el traba-

\footnotetext{
${ }^{5}$ Esta información forma parte del diagnóstico participativo realizado por el equipo de psicólogos comunitarios en La Victoria durante el año 2002, incluido en Krause, M., Velásquez, E., Ramírez, T., Turró, C., Bustamante, I. \& Torres, A. (2002). Intervención comunitaria en la población La Victoria. Informe de resultados año 2002. Santiago de Chile: Ministerio del Interior.

${ }^{6}$ La palabra cuadra se refiere a calle.
} 
jo grupal con miembros específicos de la comunidad, abarcando el período de principios de 2003 hasta mediados de 2004. El grupo se conformó a partir de representantes (hombres y mujeres) de organizaciones de distinta indole de la población La Victoria, algunos de los cuales habían formado parte del equipo mixto responsable de efectuar la Feria. Dicho grupo, se denominó Coordinadora de organizaciones sociales ${ }^{7}$ y se constituyó, apoyado por el equipo interventor, con el propósito de fortalecer las organizaciones de la población y hacer efectivo su quehacer comunitario.

\section{REFERENTES TEÓRICOS DE LA INTERVENCIÓN}

\section{La participación social como forma de actuar sobre el entorno}

Puesto que uno de los aspectos diagnosticados en la comunidad La Victoria fue el repliegue de la participación, asociado a la sensación de inseguridad ciudadana, se hizo necesario revisar los significados de aquel concepto, con el fin de integrarlos en el accionar con la comunidad, en aras al éxito de la intervención.

Si bien la participación se ha entendido de distintas formas, desde la simple colaboración, en la que los actores comparten su labor involucrándose con distintos grados de intensidad, hasta la capacidad real de un grupo de tomar decisiones (Sánchez, 1986; Gyarmati, 1992), todas ellas comparten la visión de la participación como un fenómeno, proceso u objetivo deseable para el ser humano (Montero, 1996; Sirvent, 1998). En este sentido, Sirvent (1998) afirma que una característica esencial del ser humano es la de actuar como transfor- mador del medio ambiente que lo rodea. Consecuentemente, la participación se vincula al proceso de construcción de comunidad e implica relaciones del individuo con el ecosistema en que está inmerso, generando experiencia, conocimiento y praxis, que posteriormente se incorpora a la historia de la comunidad y forma parte de la cultura (Martínez, 1998). Desde esta concepción "mesosocial" de la participación, se planteó la intervención psicológico-comunitaria; esto es, se pretendió que los actores de La Victoria fueran los responsables de provocar cierto cambio social, concretamente, el de hacer de su población un lugar más seguro donde vivir, o bien, como afirmaría Sabatini (1995), el de desarrollar mediante la participación una base de seguridad social y un sentido de pertenencia.

A un nivel más focalizado, el eje conceptual que guió la intervención fue el de la participación social, entendida como la voluntad de formar parte de un proceso colectivo y organizado de determinada manera, mediante el cual los participantes se forman y forman a otros en el manejo de conocimientos y destrezas, orientándose a propósitos, de acuerdo a sus intereses vitales y/o sociales (Sánchez, 1999). En el mismo sentido, Zimmerman y Rappaport (1988), plantean este tipo de participación como el involucramiento en alguna actividad en que el individuo participa sin percibir sueldo, en orden a lograr una meta común. Este tipo de accionar, no sólo se transforma en un vínculo necesario para el acceso a bienes y servicios, lo que favorece la satisfacción de otras necesidades de tipo psicosocial (Sirvent, 1988), sino que también se convierte en un potente aliado para predecir la calidad del ambiente físico, condiciones sociales, acceso a ser-

\footnotetext{
${ }^{7}$ A lo largo del artículo el grupo denominado Coordinadora de organizaciones sociales será referido como "Coordinadora”, con el afán de facilitar la lectura.
} 
vicios y prevención de la delincuencia (Chavis y Wandersman, 1990).

\section{El equipo de trabajo: un tipo de grupo a partir del cual participar socialmente}

Una de las formas en que la participación social se hace notoria es a través de aquellas organizaciones comunitarias que otorgan a los miembros oportunidades para desarrollar competencias, donde existen responsabilidades compartidas, se participa en la toma de decisiones, hay una atmósfera de apoyo mutuo y se realizan actividades sociales (Florin y Wandersman, 1984; Zimmerman y Rappaport, 1988; Zimmerman, 2000). A fin de lograr el tipo de participación mencionada, la intervención grupal apoyó a un grupo concreto de la población La Victoria para que se constituyera como equipo de trabajo, entendido como un sistema social altamente organizado y orientado hacia la consecución de una tarea común, en que sus miembros adoptan e intercambian roles y funciones con flexibilidad, de acuerdo a un procedimiento, y que disponen de habilidades para manejar tanto los procesos de tarea como los de mantención socioafectiva, en un clima de respeto y confianza (Morales, 1990; Kantzenback y Smith, 1995). Cabe decir que la decisión de intervenir para construir un equipo de trabajo se debió a tres razones. La primera, situarnos en una etapa avanzada de la intervención psicológico-comunitaria, después de haber trabajado con distintos actores de la comunidad y apoyarlos en las actividades realizadas, consolidando relaciones interpersonales con los pobladores, basadas en un respeto y confianza recíprocos. La segunda razón respondió al hecho de constituir un grupo con líderes y/o representantes de organizaciones sociales con historia, depositarios de cierta experiencia partici- pativa y con potencialidad para poder constituirse como un equipo sólido que pudiera dar respuesta a necesidades especificas de las organizaciones comunitarias. La tercera y última razón aludió a la importancia de que permaneciera una instancia de trabajo efectiva en la comunidad. Esto último se justificó, a su vez, por el planteamiento referido a las redes de intercambios de recursos, propuesto por Sarason y Lorentz (1979). Es decir, los recursos para satisfacer las necesidades son limitados (y más en un contexto pobre como lo es La Victoria), las personas poseen gran cantidad de recursos y potencialidades y el intercambio recíproco de los primeros mejora su utilización y eficacia. Trabajar teniendo en cuenta este planteamiento, favorecería según los autores una participación que movilizara y potenciara los recursos humanos y materiales, individuales y colectivos, con el fin de hacer del grupo un agente activo capaz de desarrollar acciones de cambio del entorno.

Puesto que conceptualmente la intervención grupal se basó en un tipo determinado de participación social, explicado previamente, metodológicamente se consideró como modelo más adecuado el de la animación sociocultural. Ésta, se basa en una de las corrientes de desarrollo comunitario, enraizada en los movimientos de Educación Popular surgidos a finales de los años sesenta en América Latina. La animación sociocultural, utiliza una serie de herramientas socio-educativas que tienen como finalidad estimular la iniciativa de las comunidades en el proceso de su propio desarrollo, además de contar con la implicación y participación de las personas y colectivos a los que va dirigida. Asimismo, tiene como finalidad la organización de y desde los sectores populares, a fin de poder transformar su realidad concreta y mejorar la vida (Bustillos y Vargas, 1999). Y esto último, tal y como señala 
Lenoir (1989), se plantea desde los procesos de responsabilización de los individuos en la gestión y dirección de sus propios recursos, elemento esencial si se considera que, para lograr el objetivo general de la intervención, el grupo concreto de la comunidad con el cual se trabajaria, debía convertirse necesariamente en equipo de trabajo.

Por último, cabe agregar que a intervención grupal tuvo en cuenta aquellos aspectos que según varios autores (García, 1983; González, 1999) son necesarios y suficientes para el buen desempeño de un equipo de trabajo (exceptuando el entorno institucional que habitualmente envuelve a este tipo de grupos):

- Un número de personas que no dificulte la interacción constructiva para llegar a acuerdos. Deben reunirse y comunicarse con facilidad y frecuencia, comprendiendo cada uno las habilidades y funciones de los otros.

- La misión es la razón de ser del equipo, lo que le da identidad y orientación a largo plazo. Debe ser el propósito del equipo comprendido por todos, constituir una aspiración más amplia que sólo metas a corto plazo.

- Debe existir una propuesta común que determine cómo trabajarán para lograr su propósito.

- Los objetivos y las metas de trabajo especificas deben ser parte del propósito del grupo, por facilitar la comunicación clara, concentrarse en los resultados y lograr pequeñas victorias que crean compromiso.

- Se debe desarrollar la mezcla de habilidades orientadas tanto al conocimiento técnico o funcional, el análisis-resolución de problemas y toma de decisión de trabajo, como también habilidades interpersonales que contribuyan a la mantención y desarrollo emocional del equipo.

- Los miembros deben ser individual y conjuntamente responsables del propósito, metas, propuesta y producto del trabajo, y para esto es necesario que conozcan con claridad sus responsabilidades individuales y conjuntas.

\section{OBJETIVOS DE INTERVENCIÓN}

Para llevar a cabo el objetivo general de la intervención grupal, apoyar al grupo de la Coordinadora de organizaciones sociales en su formación como equipo de trabajo, siendo capaz de llevar a cabo eficientemente los proyectos que se plantee, se propusieron los siguientes objetivos específicos:

1. Desarrollar el compromiso grupal.

2. Desarrollar la identidad grupal.

3. Potenciar habilidades socioemocionales: comunicación efectiva y resolución de conflictos.

4. Potenciar habilidades de tarea: planificación, toma de decisiones y distribución de roles.

\section{METODOLOGÍA DE LA INTERVENCIÓN GRUPAL}

La intervención se dividió en dos fases y se llevó a cabo por dos psicólogas de este artículo, que actuaron como facilitadoras del proceso grupal. En la primera fase de trabajo (2003) se realizó un taller de doce sesiones, de dos horas cada una, y dos jornadas de trabajo, ambas de cuatro horas. La segunda fase (2004) 
consistió en un taller de veintidós sesiones de una hora y media, y cuatro jornadas de trabajo de cuatro horas cada una.

Pensando en que el foco de la intervención era lograr que el grupo se constituyera como equipo de trabajo, se optó por una facilitación que evolucionó desde lo directivo (fijar directrices) hacia lo no directivo (utilización del reflejo de sentimientos, ideas y comportamientos grupales). De este modo, se favoreció que los participantes fueran progresivamente haciéndose cargo del proceso, reconociendo, por una parte, su estado como grupo en distintos momentos, y, por otra parte, sus potencialidades y dificultades (tanto individuales como colectivas); todo ello dirigido a que el grupo se responsabilizara de su propio funcionamiento y se independizara cada vez más de las facilitadoras. Cabe señalar, además, que las dos psicólogas tuvieron muy presente durante las dos fases de trabajo fomentar un clima de apertura y de confianza grupal, de comunicación fluida entre participantes y facilitadoras y de participación horizontal.

\section{Técnicas metodológicas utilizadas}

Las actividades realizadas con el grupo se llevaron a cabo utilizando técnicas participativas para la educación popular (análisis y discusión grupal, práctica de habilidades específicas y ani- mación grupal) derivadas de la teoría de Freire (1998), y elegidas por su carácter flexible, participativo, grupal, práctico y vivencial. Estas técnicas, a su vez, persiguieron la doble finalidad de favorecer en los participantes la adquisición de a) habilidades de tarea, para poder dar respuesta concreta a los objetivos especificos de la Coordinadora, y b) habilidades socioemocionales, que permitieran focalizarse en la interacción de los participantes, a fin de favorecer la cohesión grupal, una comunicación efectiva entre ellos y, en el caso de que aparecieran conflictos, ser capaces de afrontarlos.

Si bien se utilizaron estas técnicas metodológicas en las dos fases, se incorporaron otras dos en la segunda fase de la intervención grupal: apoyo y asesoramiento formativo a dirigentes en el rol de facilitadores durante al menos cuatro reuniones y observación no participante. En cuanto a la primera técnica, se utilizó con el objetivo de que distintos participantes adquirieran las habilidades necesarias para poder desempeñar un rol de liderazgo y contempló tres tipos de actividades: a) sesiones previas a las reuniones de la Coordinadora con una pareja de participantes, de hora y media, durante las cuales se analizaba, discutía y planificaba, junto con las facilitadoras, las actividades para la sesión, en función de los objetivos de la misma; b) realización por parte de las dos facilitadoras de una pauta ${ }^{8}$ de

\footnotetext{
${ }^{8}$ La pauta de evaluación utilizada por las facilitadoras incluía 11 comportamientos relacionados con los roles de tarea y 10 relacionados con los roles emocionales. Cada uno de estos comportamientos se evaluaba en función de una escala tipo Likert de 1 a 5 puntos. Los roles de tarea incluían los siguientes comportamientos: (1) comienza la reunión a tiempo; (2) aclara los objetivos de la presente reunión o bien pide a alguien que lo haga; (3) ofrece y pide información; (4) da y pide opiniones; (5) capacidad de sintetizar las informaciones u opiniones que se dan en el grupo; (6) controla el tiempo previsto para las actividades; (7) centra el tema hacia los objetivos planteados; (8) coordina la discusión; (9) distribuye responsabilidades; (10) Exige responsabilidades y tareas; (11) evalúa, tanto en momentos adecuados como al final de la reunión. Los roles emocionales incluían los siguientes comportamientos: (1) anima y estimula la participación; (2) concilia los desacuerdos y propone soluciones para resolverlos; (3) acepta el conflicto y las resistencias del grupo; (4) es capaz de observar las necesidades del grupo; (5) facilita la comunicación dentro del grupo; (6) sabe escuchar al otro, sin juzgar ni imponer su opinión; (7) es claro y directo en su comunicación; (8) es capaz de comprender la opinión de los otros; (9) es capaz de comprender los sentimientos de los otros; (10) establece normas de funcionamiento.
} 
evaluación en base a los comportamientos de la pareja "líder" mostrados durante la sesión y en base a los objetivos de la misma; c) entrega de retroalimentación de las facilitadoras a la pareja "lider" en dos momentos, al finalizar cada sesión y una vez terminado todo el proceso de su rol en la facilitación grupal (en base a la pauta de evaluación ya mencionada). La segunda técnica, la observación no participante, consistió en un análisis sistemático del proceso grupal y del rol de algunos participantes como facilitadores, evaluando qué aspectos de la interacción y de las habilidades relacionadas con la tarea estaban más débiles. Esta observación era utilizada, por un lado, para dar retroalimentación al grupo al final de cada reunión, con el fin de facilitar la toma de conciencia de los participantes respecto a su propio proceso grupal y así poder mejorar ciertos comportamientos y/o dinámicas. Y, por otro lado, considerar los aspectos más débiles del grupo para trabajarlos en siguientes sesiones. Por último, señalar también que en la segunda fase se implementó un recurso didáctico escrito denominado "tabla de seguimiento", con el doble objetivo de (1) realizar un seguimiento del trabajo alcanzado por el grupo y (2) enfatizar y evaluar dos aspectos, asistencia y puntualidad, fundamentales para el buen funcionamiento del grupo.

La intervención grupal consideró dos tipos de evaluaciones: de proceso y de resultados. En la primera se utilizaron dos técnicas: a) cuestionario confeccionado por las facilitadoras para las dos fases de trabajo, que incluía los aspectos positivos y mejorables del procedimiento utilizado (logros alcanzados, actividades realizadas, tiempos empleados, rol de las facilitadoras), metodologia y contenidos que debieran incorporarse en las próximas sesiones; b) técnica de observación no participante durante la segunda fase, la cual se explicó previamente. La evaluación de resultados incluyó indicadores de comportamiento (asistencia, puntualidad, tareas cumplidas por los distintos participantes) y de producto (misión, objetivos, declaración de principios y planificación del trabajo de la Coordinadora para el 2004 y el 2005) y los roles de tarea y emocionales desempeñados por los facilitadores, según el instrumento de pauta de evaluación.

\section{Contenidos trabajados con el grupo}

La primera fase de la intervención grupal desarrolló los siguientes aspectos: contrato psicológico (normas, expectativas y metodología grupal), conocimiento interpersonal, confianza interpersonal y grupal, importancia de la asistencia al grupo, elaboración de objetivos de la Coordinadora, incorporación de otros representantes organizacionales, conocimiento del accionar de las distintas organizaciones, detección de las necesidades de cada organización, significado y modo de conformación de un equipo de trabajo, habilidades de comunicación efectiva y retroalimentación, necesidad de roles diferenciados y complementarios en un equipo, elaboración de una declaración de principios (contenedora de los aspectos valóricos de su accionar) y organización de una actividad comunitaria con su consiguiente evaluación. Respecto a la segunda fase, los aspectos trabajados con el grupo fueron: integración de nuevos participantes al grupo, revisión de los objetivos y roles de la Coordinadora, compromiso individual y grupal, conocer el modo de diagnosticar y planificar actividades, identificación de los recursos individuales y colectivos, elaboración de la planificación del trabajo a realizar durante el año 2004 y 2005 y habilidades para tomar decisiones y resolver conflictos. 


\section{RESULTADOS}

A continuación se presentan los resultados, en base a los objetivos específicos planteados para la intervención grupal, y relacionándolos con los indicadores y/o técnicas de evaluación utilizados, para dar cuenta del cumplimiento de cada uno de ellos. En último lugar, se exponen los resultados vinculados con las habilidades desarrolladas por parte de algunos miembros del grupo, a los cuales el equipo interventor apoyó para que se desempeñaran como facilitadores.

El primer objetivo de la intervención, desarrollar el compromiso grupal, fue evaluado a través de tres indicadores de comportamiento: la asistencia, la puntualidad y las tareas cumplidas por los distintos participantes. En relación al primer indicador, los resultados mostraron una diferencia entre la primera y la segunda fase de trabajo. Durante la primera fase, la asistencia fue de un $76 \%$. El grupo se inició con once participantes y continuó finalmente con nueve, los cuales mostraron compromiso por el trabajo, se preocuparon de asistir regularmente, $\mathrm{y}$, en caso de no poder, se excusaron con sus compañeros de grupo y/o con las facilitadoras. En cuanto a la segunda fase, la asistencia fue del $71 \%$, iniciándose la Coordinadora con seis participantes, debido a que cinco personas del grupo inicial se retiraron, y fluctuando después entre este número y ocho participantes, debido a la integración de otros representantes durante las jornadas. Por tanto, lo que caracterizó esta segunda fase de trabajo, fue la irregularidad en la asistencia a lo largo de todo el proceso grupal, hecho que obstaculizó un buen funcionamiento grupal. La principal dificultad que ocasionó este tipo de comportamiento fue el quiebre en la cohesión grupal, ya que durante la primera fase se había logrado un nivel notable de confianza interpersonal y grupal, que disminuyó con la partida de algunos participantes y el ingreso de nuevos. En este sentido, se tuvo que reiniciar el trabajo de conformación de grupo, para conseguir nuevamente un clima adecuado de confianza. Este hecho enlenteció el trabajo previsto para la segunda fase, por tener que aplazarse los objetivos planificados para las primeras sesiones en pro del logro de un buen clima grupal.

Los resultados obtenidos del segundo indicador, la puntualidad, evidenciaron que fue uno de los aspectos dificiles de cumplir por el grupo. Durante las primeras sesiones del taller de 2003, algunos participantes llegaban entre quince y treinta minutos más tarde de la hora de inicio. Este retraso era debido a la demora fluctuante de los transportes públicos, ya que los participantes llegaban de su trabajo, realizado en zonas muy alejadas de La Victoria. Tan solo una participante demoraba por no tener integrado el respeto por la puntualidad. Al ser la presencia de todos esencial durante las primeras sesiones, por la necesidad de favorecer la cohesión grupal y el conocimiento interpersonal, se optó por iniciar la sesión una vez todos los participantes hubieran llegado. Esto producía que parte de lo planificado para una sesión concreta no pudiera realizarse y se tuviera que desplazar para la siguiente. A pesar del énfasis expresado por las facilitadoras al grupo sobre el respeto por la puntualidad, y que los participantes lo señalaran como norma de funcionamiento grupal, sólo se cumplió medianamente. La mayor puntualidad lograda fueron quince minutos después de la hora de inicio que el grupo había fijado. Esta dificultad se discutió con el grupo antes de iniciar la segunda fase de trabajo, acordando los miembros de la Coordinadora con las facilitadoras, empezar media hora después, estableciéndose sesiones de hora y media, y realizar jornadas de mayor duración. Esta medida, junto con 
"la tabla de seguimiento", donde se anotaba la hora de inicio y cierre de las sesiones, fue decisiva para que la puntualidad fuera respetada por todos.

Los resultados del último indicador, tareas cumplidas por los miembros, incluyeron tanto las tareas realizadas para el buen funcionamiento grupal, como las desempeñadas para el logro de la $2^{\text {a }}$ Feria de organizaciones sociales. En relación a las del primer tipo, se pidió al grupo que efectuara cinco tareas: (1) elaboración de un resumen con los contenidos trabajados en cada sesión, clima grupal y resultados obtenidos, realizado cada vez por un participante y leído al principio de la sesión. Dicho resumen, pretendía ser una memoria de lo que iba aconteciendo a lo largo de las sesiones, a fin de que el grupo fuera conciente de su propio proceso. Cabe señalar que, de la totalidad de miembros de la Coordinadora, tan solo dos no realizaron la tarea, por no asistir a las dos sesiones donde tenian que leer el resumen. El resto de participantes cumplió con la tarea plenamente, además de expresar su utilidad como medio de comprensión y profundización de lo acontecido en el grupo; (2) preparación de una exposición de la organización a la cual representaban, respondiendo a varios contenidos que ellos mismos seleccionaron, fruto del trabajo grupal. Todos los participantes cumplieron con la tarea, exponiendo su organización a la Coordinadora entre la quinta, sexta y séptima sesión, utilizando en algún caso material de apoyo visual; (3) detección por parte de cada uno de los participantes de las necesidades percibidas por sus respectivas organizaciones y llevarlas por escrito a una jornada determinada, para poder derivar de ellas los principales objetivos que debía tener la Coordinadora. Si bien la tarea se realizó, no se cumplió en el plazo fijado, sino dos sesiones posteriores a la jornada; (4) y (5) designación de tareas concretas a miembros específicos del grupo, en función de la distribución de roles realizada en la segunda fase de trabajo grupal. Así, la primera de estas tareas, cumplidas plenamente, se relacionó con el rol de secretaria y consistió en anotar los resultados y los acuerdos llegados en las sesiones, así como leerlos al inicio de cada una de éstas. La quinta tarea se asignó a las tres parejas que actuaron como facilitadores y consistió en la preparación de las actividades a realizar para cada sesión, previamente al inicio de cada una. Esta tarea fue la que menos se cumplió, pues las parejas de facilitadores 0 bien llegaban tarde a la preparación de las sesión con el equipo interventor, o bien llegaban puntualmente pero sin haber preparado entre ellos/as la sesión.

Especialmente importante para evaluar el compromiso grupal fue la organización y realización de una actividad comunitaria realizada por la Coordinadora durante buena parte de la primera etapa de trabajo, en el 2003: la $2^{\text {a }}$ Feria de organizaciones sociales. Ésta tuvo su antecedente en el año 2002, con la realización de la $1^{a}$ Feria de organizaciones sociales, la cual se explicó brevemente en una sección previa del artículo. De modo similar a ésta, la $2^{\mathrm{a}}$ Feria consistió en una actividad callejera en que las distintas organizaciones mostraban su quehacer a través de stands y paneles, donde exponian sus objetivos, actividades y productos. Asimismo, se contó con un escenario desde el cual se presentaron diversos actos artístico-culturales. Para llevar a cabo la actividad, se realizó una distribución de tareas entre los miembros de la Coordinadora en función de las motivaciones y capacidades de las personas. Pese a que hubo un avance por parte de algunos miembros en cuanto a asumir sus tareas, realizándolas a tiempo, otros no las realizaron, lo que provocó, por una parte, malestar en el 
grupo, por no poderse realizar algunas actividades previstas, y, por otra, la exigencia de quienes sí cumplieron con las tareas asignadas. Así, la $2^{a}$ Feria de organizaciones sociales contó con la participación de trece organizaciones, incluyendo tanto a organizaciones que formaban parte de la Coordinadora como otras de La Victoria. Respecto a la evaluación que la Coordinadora realizó de la actividad, el grupo destacó como aspectos positivos: la elaboración y exhibición de pendones con la información de la Coordinadora, ubicados al inicio de la calle donde se instaló la Feria, difusión de las organizaciones que la constituian, así como su Declaración de Principios, paneles con información de distintas organizaciones sociales de La Victoria, con contenido claro y, por último, la incorporación en la actividad de dos organizaciones no participantes en la Feria del año pasado.

El segundo objetivo de la intervención, desarrollar la identidad grupal, se evaluó con tres indicadores de producto; esto es, misión, objetivos y declaración de principios de la Coordinadora. Luego de un largo proceso de detección de necesidades personales y organizacionales, se obtuvo una revisión acabada de los dos primeros indicadores. Así, la Coordinadora logró un conocimiento claro, compartido y aceptado de su misión: fortalecer las organizaciones a nivel interno. En cuanto a la definición de los objetivos, ésta fue compleja por la ambiciosa voluntad de parte del grupo de trabajar a nivel comunitario. Con tal de simplificar y hacer realista la tarea de la Coordinadora, se consideró la elaboración de objetivos a partir de la detección de necesidades que cada participante realizó de su respectiva organización. Así, surgieron cinco objetivos: (1) capacitar a las organizaciones en los temas demandados: realización de proyectos, educación civica, difusión de la organiza- ción y resolución de conflictos; (2) apoyar con recursos humanos a las distintas organizaciones en las actividades comunitarias que realicen; (3) buscar espacios físicos para realizar actividades; (4) realizar actividades para generar recursos económicos y (5) mejorar los canales de comunicación entre la Coordinadora $\mathrm{y}$ las organizaciones sociales externas a ella. Por último, a partir de la propuesta realizada por uno de los participantes al grupo, se desarrolló una declaración de principios, con el fin de abarcar los aspectos valóricos que guiaban el quehacer de la Coordinadora. La propuesta fue acogida por el grupo y se plasmó en una declaración que tenía como ejes la solidaridad, la identidad como "victorianos" y el bienestar psicosocial de los pobladores.

El tercer objetivo, orientado a potenciar las habilidades socioemocionales, específicamente de resolución de conflictos y de comunicación efectiva, se valoró a través del cuestionario de evaluación de proceso y de la observación no participante por parte de las facilitadoras. En relación a los resultados obtenidos del cuestionario, los participantes mencionaron en la sexta sesión del taller del 2003 lo siguiente: existencia de mayor confianza interpersonal respecto al inicio del taller, aprendizaje de capacidad autocrítica respecto al comportamiento con y para el grupo y mayor tolerancia a las diferencias valóricas e ideológicas de los miembros. Respecto a la segunda fase de trabajo, los participantes señalaron en relación al año anterior que habia más participación de todos los miembros de la Coordinadora, basada en un mayor diálogo y discusión entre ellos. Asimismo, también indicaron que existía una mayor capacidad de expresar los sentimientos, así como una expresión más libre de sus reacciones negativas. Esta evaluación de los participantes del proceso grupal durante el año 2004, se 
complementó con algunos aspectos obtenidos de la técnica de observación no participante. Respecto a la habilidad de comunicación efectiva, los participantes (1) se escuchaban sin interrumpirse, (2) la escucha entre ellos era con respeto, (3) la conversación era más argumentada que al inicio del trabajo del 2003 y (4) exponian distintos puntos de vista. En cuanto a la habilidad para resolver conflictos se observó que (1) había dificultad en enfrentar los conflictos, en el sentido de tener miedo a confrontar, (2) al aparecer un conflicto, la tendencia del grupo era a la minimización o incluso a la negación y (3) planteamiento más directo de los conflictos paulatinamente, en el sentido de atreverse a emitir opiniones discordantes.

El cuarto y último objetivo, potenciar habilidades relacionadas con la tarea, concretamente, de planificación, distribución de roles y toma de decisiones, fue evaluado a partir de la organización de la actividad comunitaria " $2^{\mathrm{a}}$ Feria de organizaciones sociales", el indicador de producto obtenido de la planificación del trabajo para los años 2004 y 2005 y los resultados de la observación no participante. Respecto a la habilidad de planificación, se observó que la Coordinadora inicialmente tendía más al activismo que al trabajo en base a necesidades y objetivos reales. Es decir, por una parte, existía poca reflexión en torno a los objetivos de las actividades que planteaba, orientadas a reunir las organizaciones sociales, con un propósito basado en la tradición, que incluso referían con sus propias palabras: "hacer lo que siempre hemos hecho", o bien para mostrar que éstas seguian vivas. Por otra parte, las actividades se realizaban en base a la improvisación y a la "buena voluntad". Esto dos aspectos tuvieron como efecto que las facilitadoras propusieran al grupo priorizar la detección de necesidades, a nivel de las organizaciones socia- les que cada miembro de la Coordinadora representaba, como criterio de realidad y en aras a definir objetivos claros y planificar acciones.

La Coordinadora, gracias a la puesta en práctica de la planificación para realizar la $2^{\text {a }}$ Feria de organizaciones sociales, se dio cuenta del aporte que esta herramienta podia suponer para sus futuras actividades, en cuanto a mejorar el resultado y calidad de éstas. En el mismo sentido, la planificación del trabajo para los años 2004 y 2005 fue un logro importante, en tanto permitió establecer objetivos a mediano plazo, consolidó los objetivos de la Coordinadora en forma de actividades concretas y definió un cronograma para cada año, con tareas y responsables, susceptibles de estar sujetas a revisión y evaluación para lograr su cumplimiento.

Respecto a la capacidad de distribuir roles, el equipo, de manera similar a como llevaba a término la planificación durante la primera etapa de trabajo, actuaba con una suerte de inercia. Es decir, determinaba los encargados de algunas tareas sin asegurar responsabilidad ni factibilidad real, lo que muchas veces derivaba en tareas no cumplidas, improvisaciones de último momento y confusiones en relación al grado de responsabilización del encargado/a de la tarea o bien en qué consistía ésta. Junto con el proceso de aprendizaje en planificación, la Coordinadora fue comprendiendo a partir del diseño, organización y realización de la $2^{\underline{a}}$ Feria de organizaciones sociales, la necesidad de definir con antelación los roles que cada uno desempeñaría en una actividad, así como la importancia de asumir y exigir responsabilidad frente a las tareas asignadas, para que éstas fueran ejecutadas de manera efectiva. Una de las actividades que fue realizada en una sesión grupal mientras se estaba organizando la $2^{\text {a }}$ 
Feria, consistió en identificar a nivel individual las competencias que cada miembro tenía en función de los requerimientos de los distintos roles necesarios para llevar a cabo dicha actividad comunitaria. Gracias a este trabajo, el grupo tomó conciencia sobre la importancia de predefinir los requisitos necesarios para cada rol, así como reconocer las competencias individuales más adecuadas para su óptima realización.

En cuanto a la toma de decisiones, ésta fue una de las habilidades más complejas de trabajar. Al grupo le costó diferenciar entre facilitar la toma de decisiones e imponer autoritariamente una decisión; si bien los participantes entendían la relevancia del diálogo, les costaba concebir la riqueza de expresar opiniones divergentes en pro de la búsqueda de opciones creativas, a partir de las cuales llegar a consenso. Entonces, la Coordinadora al principio tendía a mantener conversaciones que podían alargarse, incluso durante semanas, sin llegar a acuerdos ni decisiones, las que finalmente eran tomadas, por la urgencia de tiempo, por una persona. Ahora bien, en otras ocasiones se producía un funcionamiento contrario, es decir, al existir cierto temor en el grupo de ser autoritario, el resultado final era un comportamiento grupal basado en no tomar decisiones. El desarrollo de esta habilidad fue especialmente abordada a partir de la retroalimentación individual y grupal. Como resultado de esta técnica cabe señalar que, si bien no se alcanzó el nivel esperado, es decir, que las decisiones se tomaran de común acuerdo luego de una discusión que involucrara distintas miradas al problema, se mejoró en dos sentidos: la responsabilidad de ciertos líderes en asumir la toma de decisiones en los tiempos correspondientes y la concreción del tema central de las discusiones como método para facilitar los acuerdos grupales.
El último aspecto a señalar en esta sección de resultados son los comportamientos más desarrollados por los seis miembros de la Coordinadora (un hombre y cicno mujeres), en su rol como facilitadores. Para ello, se utilizó en la primera sesión donde se desempeñaron como facilitadores, la pauta de evaluación que incluia los roles de tarea y emocionales, resultado que fijó el punto de partida a partir del cual poder entregar una retroalimentación de su estado de avance una vez finalizadas todas las sesiones en que algunos miembros asumían un rol de facilitación. Así, por un lado, cabe señalar que tres de los participantes potenciaron roles de orientación emocional, en menoscabo a los de tarea, destacando de los primeros la capacidad de comprender las opiniones y sentimientos de los otros, generar un clima de trabajo grato, ser empático y asertivo y tener sentido del humor. Por otro lado, dos miembros distintos a los indicados previamente, mostraron su fortaleza en los roles de tarea, específicamente, en la capacidad para centrar el tema y seguir los objetivos propuestos para la reunión, controlar el tiempo de las actividades realizadas, pedir al grupo que se responsabilizara de ciertas tareas, retomar los temas $\mathrm{u}$ objetivos cuando el grupo se dispersaba y generar confianza y seguridad en el grupo por cuanto mostraban conocer bien las actividades. A fin de que estos cinco participantes desarrollaran los roles que tenían más débiles, se los retroalimentó sesión tras sesión, entregándoles por escrito, por parte del equipo interventor, las áreas a mejorar. Ahora bien, cabe señalar que la facilitación efectuada en pareja fue positiva, en tanto se produjo coordinación y complementación de roles entre los dos facilitadores; es decir, si uno de ellos mostraba mayor fortaleza en el rol de orientación emocional y dificultad en la orientación a la tarea, el otro facilitador se desempeñaba en este rol, y viceversa, teniendo 
como efecto la presencia en el grupo de los roles emocionales y de tarea. Por último, debe destacarse que una de las participantes, potenció como facilitadora de igual manera los dos tipos de roles, destacándose entre sus capacidades las de saber escuchar, impregnar de energia al grupo y ser capaz de enfrentar los conflictos.

\section{CONCLUSIONES}

Se presenta a continuación la valoración general de la intervención grupal, exponiendo primeramente los logros, asî como las dificultades en relación a ésta $\mathrm{y}$, finalmente, algunas reflexiones sobre la experiencia, las cuales pueden iluminar algunas intervenciones comunitarias futuras.

En relación a esta experiencia profesional, cabe mencionar, de modo general, tres logros. El primero se refiere a la identidad grupal desarrollada por los miembros de la Coordinadora. Estos consiguieron internalizar su razón de ser como grupo, teniendo clara su finalidad y su relación con la misión y valores de la organización, así como los objetivos propios que le daban sentido a la interacción e interdependencia grupal. En este sentido, la Coordinadora pasó a ser un referente poblacional respecto a las organizaciones sociales y a la implementación de actividades comunitarias, fomentando la ocupación de los espacios públicos y generando canales de comunicación entre las organizaciones y los pobladores. Esto último, conseguido a través de la realización de la actividad comunitaria " 2 " Feria de organizaciones sociales". Asimismo, ésta significó el primer paso del quehacer de la Coordinadora como equipo autónomo, sin que el equipo interventor tuviera la responsabilidad organizativa, además de ser capaz de llevar a cabo un proyecto comunita- rio. El tercer logro dice relación con la adquisición de habilidades interpersonales, tanto de orientación emocional como de tarea, explicadas en detalle en la sección de resultados, así como conseguir un adecuado compromiso e identidad grupales, aspectos esenciales para que un equipo pueda trabajar de manera colaborativa y orientada a objetivos específicos.

Respecto a las dificultades del trabajo grupal, la primera que se debió enfrentar fue la discrepancia entre la percepción que tenían los pobladores sobre su "sí mismo" y su estado real. Es decir, los pobladores de La Victoria se definían a sí mismos como una comunidad con mucha fuerza y participación, capaces de organizarse y generar recursos desde la carencia y con fuerte "sentido de comunidad". Sin embargo, el trabajo cotidiano nos hizo ver otra realidad, la de un grupo con participación, pero a mucha menor escala de la percibida por ellos, debida esta situación sobre todo al microtráfico y la desesperanza, esta última producto de una transición democrática que heredaba, entre otros, valores económicos neoliberales, además de no cumplir el nuevo gobierno con las expectativas de equidad y mayor calidad de vida para todos los chilenos. Estas atribuciones las habian expresado los miembros de la Coordinadora, quienes, cabe no olvidar, representaban a distintas organizaciones sociales consolidadas de La Victoria. En coherencia con lo planteado, la discrepancia mencionada en la percepción como pobladores entorpeció el funcionamiento grupal, puesto que la fuerza que el "pasado" impregnaba en los participantes de la Coordinadora, dificultaba re-construir una nueva realidad organizacional presente. A nivel de equipo interventor, implicó también realizar un re-ajuste entre las expectativas optimistas en relación a la existencia en el grupo de cierta base de habilidades y 
actitudes en relación a la participación y la situación real.

La segunda dificultad en la experiencia grupal realizada fue lograr una asistencia regular por parte de los participantes. Durante el proceso observamos lo complicado que fue para el grupo incorporar esta norma, lo que pudo explicarse por distintos motivos: llegada tarde del trabajo y cansancio, hábito de impuntualidad arraigado, sobrecarga en otras actividades de su organización 0 , simplemente, atribuciones externas (por ejemplo, llovia y se suponía que no había reunión de grupo). Ahora bien, todos estos aspectos eran aristas de un mismo comportamiento, el cual podía explicarse por una cierta dinámica grupal. Esto es, generalmente los grupos tienden a ser conservadores en su funcionamiento, en el sentido que, un comportamiento ya instalado, es muy dificil de cambiar, debido a que los miembros del grupo tienden a actuar económicamente (realizar la conducta que genere menor gasto energético). Esto implica actuar bajo supuestos, sin contrastarlos con los hechos (González, 1999). Cambiar un comportamiento, desde esta perspectiva, significaba tomar conciencia de las dificultades que generaba, lo que implicaba un mayor esfuerzo y, por tanto, un mayor gasto energético que el comportamiento ya instalado.

La última dificultad con la que se enfrentó el equipo interventor fue desarrollar en el grupo la capacidad de resolución de conflictos. Resulta interesante puntualizar que, la tendencia grupal era la negación o minimización de los conflictos, lo que impedía su enfrentamiento y resolución. Durante el proceso se implementaron distintas estrategias para trabajar dicha habilidad, a través de técnicas o conversándolo directamente, sin embargo, no hubo grandes cambios. Al respecto, se hipotetizó que el comportamiento de negación al conflicto podría relacionarse con una de las secuelas sociales provocadas por los años de dictadura militar que vivió el país, donde el rechazo y el miedo al conflicto eran dos procederes recurrentes.

Respecto a las reflexiones surgidas a raíz de la experiencia de trabajo comunitario presentada, un aspecto a resaltar, dice relación con el proceso de inserción del equipo de profesionales en la comunidad, en dos direcciones concretas: la de respetar los tiempos y estilos de trabajo de la población y la de integrar el rol técnico de los profesionales. Todos ellos, elementos que implicaban partir de una postura de intervención que valoraba los recursos de la comunidad frente a sus carencias. En cuanto a la inserción en La Victoria, fue necesario hacerlo a través de un acercamiento paulatino, con una actitud de apertura y de escucha de las necesidades poblacionales, rescatando las experiencias y sabidurías de la comunidad y, especialmente, teniendo en cuenta que la población donde trabajamos era poseedora de una historia participativa. Estas actitudes fueron útiles tanto para resquebrajar las resistencias que toda comunidad tiene ante un equipo interventor externo, como para ir entrelazando una relación de confianza. En cuanto al rol profesional del equipo interventor, se tuvo especial cuidado durante toda la experiencia, del aporte conjunto entre equipo y pobladores en cuanto a conocimientos y habilidades, de tal manera que se favorecía una potenciación y enriquecimiento de ambos.

Otro aspecto importante de rescatar es la relevancia que cobró en el quehacer comunitario la discusión con la totalidad del equipo interventor. En este sentido, los espacios de reflexión de las facilitadoras grupales, además de las reuniones con el equipo completo, permitieron observar, desde dentro de la experiencia grupal y desde una postura 
más distante a ésta, el proceso, facilitando la toma de decisiones en relación a las mejores alternativas a seguir para el logro de los objetivos propuestos. Tal y como se explicó a lo largo del artículo esta experiencia profesional, la metodología utilizada y los objetivos abordados implicaban fomentar directamente la participación de un colectivo concreto de la comunidad, partiendo siempre desde las preocupaciones, necesidades y fortalezas propias del grupo. Coincidente con lo planteado, Gento (1994) señala que la intervención puede ser vista como la intervención de personas 0 grupos en la discusión y toma de deci- siones, compartiendo para ello trabajos específicos, lo que implica asumir parte del poder o del ejercicio del mismo. Es por ello que el equipo interventor tuvo en cuenta que la importancia de trabajar desde y para la participación implicaba que el grupo de la Coordinadora de organizaciones sociales se involucrara activamente, desde el comienzo, en el proceso de desarrollo de la intervención. tomando conciencia de la situación existente y de sus causas, así como de las acciones que pudieran conducir a la superación de la situación, aspectos pudieron observarse en el desarrollo de la intervención expuesta.

\section{BIBLIOGRAFÍA}

Bustillos, G. \& Vargas, L. (1999). Técnicas participativas para la educación popular (Tomo II). Madrid: Editorial

Chalom, M., Léonard, L., Vanderschueren, F., y Vézina, C. (2001). Seguridad ciudadana, participación social y buen gobierno: el papel de la policía Santiago: Edicioanes Sur

Chavis, D. M. y Wandersman, A. (1990). Sense of community in the urban environtment: A catalyst for participation and community development. American Journal of Community Psychology, $18,55-81$.

Cortés, A. (2003). Rescatando memoria. Guía introductoria al estudio de la población La Victoria: la toma de terrenos [En red]. Disponible en: http://www.jjcc.cl

Florin, P. y Wandersman, A. (1984). Cognitive social learning and participation in community development. American Journal of Community Psychology, 16, 808-830.

Freire, P. (1998). La educación como práctica de la libertad (46 ${ }^{\mathrm{a}}$ ed.). Madrid: Siglo XXI de España Editores.

García, M. (1983). El trabajo en equipo. México: Fondo Educativo Interamericano.

Gento, S. (1994). Participación en la gestión educativa. Madrid: Santillana. 
Una experiencia en Chile de trabajo grupal con dirigentes de organizaciones sociales

González, P. (1999). Psicología de los grupos: Teoría y Aplicación. Ed. Sintesis: España.

Gotzens, P. (1993). Programa de actuación sobre la problemática de las drogodependencias, a través de la acción comunitaria, en un barrio periférico de la ciudad de Valencia. En G. Musitu, E. Borjano, E. Gracia y J. R. Bueno (Eds.), Intervención psicosocial. Programas y experiencias (pp. 6174). Madrid: Editorial Popular.

Gyarmati, G. (1992). Salud, poder y democracia: hacia una teoría de la atención primaria. Santiago de Chile: Instituto de Sociologia. Pontificia Universidad Católica de Chile.

Kantzenback, J. y Smith (1995). La sabiduría de los equipos. México: CECSA.

Krause, M., Velásquez, E., Ramírez, T., Turró, C., Bustamante, I. y Torres, A. (2002). Intervención comunitaria en la población La Victoria. Informe de resultados año 2002. Santiago de Chile: Ministerio del Interior.

Krause, M. y Jaramillo, A. (1998). Intervenciones psicológico-comunitarias en Santiago de Chile. Santiago: Pontificia Universidad Católica de Chile, Facultad de Ciencias Sociales.

Lenoir, R. (1989). La notion de développement. Foi et Développement, 175-176

Martínez, M. L. (1998). Psicologia Comunitaria e intervención en comunidades. En M Krause y A. Jaramillo (Eds.), Intervenciones psicológico-comunitarias en Santiago de Chile (pp. 17-47). Santiago: Pontifica Universidad Católica de Chile, Facultad de Ciencias Sociales.

Montero, M. (1996). La participación. Significa- do, alcances y limites. En E. Hernández (Eds.), Participación. Ámbitos, retos y perspectivas (pp. 7-20). Caracas: CESAP.

Morales, M. (1990). Desarrollo grupal y liderazgo efectivo (Publicación interna). Santiago: TOP consultores, S.A.

Paiva, M. (1989). Pasado: Victoria del presente. Santiago: Salud Poblacional.

Sabatini, F. (1995). Barrio y participación. Mujeres Pobladoras de Santiago. Santiago: Ediciones Sur.

Sánchez, M. (1986). Metodología y práctica de la participación. Madrid: Editorial Popular.

Sánchez, E. (1999). Todos para todos: la continuidad de la participación comunitaria. Psykhe, 9(1), 135-144.

Sarason, S. y Lorentz, E. (1979). The challenge of the resource exchange network. San Francisco: Jossey-Bass.

Sirvent, M. T. (1988). Investigación participativa, mitos, metodología y técnicas. Seminario de metodología de la investigación social. Corrientes.

Sirvent, $\mathrm{M}^{\mathrm{a}} \mathrm{T}$. (1998). Los diferentes modos de operar en investigación social. Buenos Aires: Universidad de Buenos Aires.

Zimmerman, M. y Rappaport, J. (1988) Citizen participation, perceived control, and psychological empowerment. American Journal of Community Psychology, 16(5), 725-760.

Zimmerman, M. (2000). Empowerment theory. En J. Rappaport y E. Seidman (Eds.), Handbook of Community Psychology. 